\title{
Insights into the Binding Mechanism of Ascorbic Acid and Violaxanthin with Violaxanthin De-Epoxidase (VDE) and Chlorophycean Violaxanthin De-Epoxidase (CVDE) Enzymes: Docking, Molecular Dynamics, and Free Energy Analysis
}

\section{Satyaranjan Biswal, Parth Sarthi Sen Gupta, Haamid Rasool Bhat, and Malay Kumar Rana*}

Department of Chemical Sciences, Indian Institute of Science Education and Research (IISER) Berhampur, 760010 Odisha.

\begin{abstract}
Photosynthetic organisms have evolved to work under low and high lights in photoprotection, acting as a scavenger of reactive oxygen species. The light dependent xanthophyll cycle involved in this process is performed by a key enzyme (present in the thylakoid lumen) Violaxanthin DeEpoxidase (VDE) in the presence of violaxanthin and ascorbic acid substrates. Phylogenetically, VDE is found to be connected with an ancestral enzyme Chlorophycean Violaxanthin DeEpoxidase (CVDE) present in the green algae on the stromal side of the thylakoid membrane. However, the structure and functions of CVDE were not known. In search of functional similarities involving this cycle, the structure, binding conformation, stability, and interaction mechanism of CVDE are explored with the two substrates in comparison to VDE. The structure of CVDE was determined by homology modeling and validated. In-silico docking (of first-principles-optimized substrates) revealed it has a larger catalytic domain than VDE. A thorough analysis of the binding affinity and stability of four enzyme-substrate complexes are performed by computing free energies and its decomposition, the root-mean-square deviation (RMSD) and fluctuation (RMSF), the radius of gyration, salt-bridge and hydrogen bonding interactions in molecular dynamics. Based on these, violaxanthin interacts with CVDE to the similar extent as that of VDE, hence its role is expected to be the same for both the enzymes. On the contrary, ascorbic acid has a weaker interaction with CVDE than VDE. As these interactions drive epoxidation or de-epoxidation process in the xanthophyll cycle, it immediately discerns that either ascorbic acid does not take part in de-epoxidation or this process requires a different cofactor because of the weaker interaction of ascorbic acid with CVDE in comparison to VDE.
\end{abstract}


Keywords: Xanthophyll Cycle; VDE; CVDE; DFT; Molecular Dynamics; Free Energy

\section{Introduction}

Without photosynethesis, which is triggered by light, plants and animals can barely exist on the planet. Due to varitions of light (both quantity and quality), photosynthetic organisms have developed different strategies to maintain a fine balance between light harvesting photochemistry and photoprotection[Külheim et al., 2002]. The photoprotection mechanism consists with the dissipation of excess light energy in the form of heat, thus avoiding photo-oxidative damages caused by the reactive oxygen species (ROS)[Siefermann-Harms, 1985; Stransky and Hager, 1970]. When the limit of light intensity-exceeds, the photosynthetic light harvesting complex is regulated rather by non-photochemical quenching (NPQ) mechanisms responsible for dissipating excess absorbed light as heat[Foyer, 2018; Müller et al., 2001; Niyogi and Truong, 2013; Ruban et al., 2012]. The most exclusively and intensively investigated component of NPQ is the high energy state quenching ( $\mathrm{qE}$ ), which leads to dropping of $\mathrm{pH}$ in thylakoid lumen and acidification in excess light[Niyogi and Truong, 2013]. Among all, the dissipation of heat by carotenoids represents the most important photoprotective mechanism actively functioning in thylakoid membranes of plants and algae[Briantais et al., 1979; Britton, 1976; Bungard et al., 1999]. This process is accomplished by a cycle called "xanthophyll cycle" by interconverting violaxanthin carotenoid to antheraxanthin to zeaxanthin and vice-versa, respectively, at high light, low $\mathrm{pH}$ and low light, high $\mathrm{pH}$ conditions. Zeaxanthin is an antioxidant which scavenges reactive singlet oxygens by quenching of the excited singlet and triplet states of chlorophyll thereby accelerating the photoprotection capacity[Casper-Lindley and Björkman, 1998; Coesel et al., 2008; Lunch et al., 2013; Niyogi et al., 1997; Niyogi et al., 1998]. When light-driven proton translocation rate across the thylakoid membrane exceeds the dissipation rate of the proton gradient by ATPase leads to a reduction in $\mathrm{pH}$ in the thylakoid lumen activating Violaxanthin De-Epoxidase (VDE)[Baroli et al., 2003; Quaas et al., 2015; Yokthongwattana and Melis, 2006]. Activation of VDE occurs with a conformational change [Baroli et al., 2003; Bratt et al., 1995; Hager and Holocher, 1994; Morosinotto et al., 2002] and its association with the thylakoid membrane with the involvement of substrate violaxanthin [Bratt et al., 1995; Hager and Holocher, 1994; Morosinotto et al., 2002]. The role of zeaxanthin in photoprotection mechanism is vital as a mutant form of VDE shows an 
increased susceptibility to high light and reduced fitness in natural conditions due to de-excitation mechanisim[Külheim et al., 2002]. Whereas in low light conditions, the process gets reversed and zeaxanthin is converted back to violaxanthin by the stromal enzyme Zeaxanthin Epoxidase (ZE). [Bugos et al., 1998; Hieber et al., 2000]

A mutational variant (defective) of NPQ1 gene has been identified in the unicellular green alga Chlamydomonas reinhardtii and the Arabidopsis thaliana, which plays a significant role in the process of xanthophyll cycle and qE[Bugos et al., 1998; Hieber et al., 2000; Niyogi et al., 1998]. This NPQ1 mutant gene in Arabidopsis thaliana is unable to continue the process of VDE catalyzed conversion of violaxanthin (Vio) to zeaxanthin (Zea) in excessive intensity of light. On the other hand, in case of Chlamydomonas, the precise role of NPQ1 mutant in xanthophyll cycle is yet to be clearly understood. In case of Chlamydomonas genome, recent evidences suggest that it lacks a distinct orthologue of the VDE gene in plants and other algae [Anwaruzzaman et al., 2004; Hieber et al., 2000].

Recently, a new type of VDE gene has been found in Chlamydomonas reinhardtiien present at the stromal side of the thylakoid membrane, which helps translate a special type of VDE protein called Chlamydomonas CVDE (CrCVDE) protein. The evolutionary origins of plant-type VDE and CVDE are clearly distinct [Li et al., 2016]. For CVDE, CruP and CruA, there presents a common ancestor. CruA is known to be involved in bacterial carotenoid biosynthesis as a lycopene cyclase, whereas CruP is a paralogue of CruA, widely found in oxygenic photosynthetic living organisms[Bradbury et al., 2012]. Zhirong Li et al. hypothesized based on phylogenetic analysis that CVDE evolved by duplication of CruP from the ancestor of green algae and plants. They also categorized Chlamydomonas CVDE as a special type of protein which has been lost earlier. Billion years ago, it might be seen in some ancient clades of photosynthetic organisms such as green algae[Merchant et al., 2007], which effectively adapts itself to increase the photosynthetic efficiency and productivity.

Both VDE and ZE have been recognized as lipocalins, i.e. a member of multigenic protein family consisting of conserved structural pattern with a 8-strand b-barrel [Bugos et al., 1998; Hieber et al., 2000]. Lipocalin proteins are mostly involved in transporting small hydrophobic molecules such as steroids, bilins, retinoids, and lipids; one of the few lipocalins also exhibits catalytic 
activity in case of VDE and ZE[Hieber et al., 2000]. The structure of the VDE lipocalin domains (VDEcd and VDE central domain) possesses a structural pattern typical of that of a multigenic family, and also evidences a $\mathrm{pH}$-dependent conformational change associated with protein activation that induces dimerization, allowing both Violaxanthin rings to react at the same time[Arnoux et al., 2009]. VDE has Cysteine-rich and Glutamate-rich two additional domains, as evident with no clear similarity with other proteins due to their peculiar amino acid composition[Bugos et al., 1998; Bugos and Yamamoto, 1996; Hieber et al., 2000; Morosinotto et al., 2002]. Both domains are found to be essential for protein activities, with their deletion leading to protein damage and inactivation of VDE [Bugos and Yamamoto, 1996]. While the Glutaminerich domain has been suggested to be involved in protein association to the thylakoids membrane, the Cystine-rich one has unknown functional roles [Bugos and Yamamoto, 1996; Hieber et al., 2002]. While VDE is fairly known, studies on CVDE barely exist. There is no information about the sequence, structure, and functions reported for such enzyme giving any molecular level insights. As there is a phylogenetic connection between CVDE and VDE, it is instructive to predict their structural and functional similarities, exploring their interactions and binding mechanisms with substrates.

The aim of the present study is to investigate the molecular characteristics of the newly discovered de-epoxidase enzyme on the stromal side of the thylokoid membrane of Chlamydomonas CVDE (CrCVDE) protein and also inspect its roles in the xanthophyll cycle. A comparative assessment between VDE and CVDE is performed. The three dimensional (3-D) structural details of CVDE were deduced using a protocol that includes multiple-template homology modeling, protein threading ab intio modeling, and other Bioinformatics tools followed by structure validation. Insilico docking analysis of both enzymes CVDE and VDE were carried out with violaxanthin and ascorbic acid substrates to determine binding sites. Additional electronic structure information of the substrates were also provided. Post-molecular dynamics analysis, such as the root-mean-square deviation (RMSD) and fluctuation (RMSF), salt-bridges, h-bonds, key residues, principal components (PC) and free energies, demonstrates that both enzymes interact with violaxanthin to a similar extent, however, the interaction of ascorbic acid with CVDE is weaker than VDE. As the interaction of violaxanthin and ascorbic acid with VDE/CVDE guides the epoxidation or deepoxidation process of the xanthophyll cycle, the latter substrate may not be required by CVDE or has trivial roles in the algae owing to the weak interaction. ${ }^{31}$ 


\section{Materials and Methods}

\subsection{Sequence Analyses and Homology Modeling}

The amino acid sequence of CVDE was retrieved from the protein sequence database UniPortKb with UniPortKb-ID: A0A2K3DUD0 (https://www.uniprot.org). The functional domains of both VDE and CVDE were identified by using Conserved Domains Database (CDD). BLASTp tool of NCBI was used for identifying a suitable template for the computational modeling of CVDE [Marchler-Bauer et al., 2010]. Multiple sequence alignment between template and target was performed by MultAlin tool (http://www. sacs.ucsf.edu/cgi-bin/multalin.py) and ESPript[Altschul et al., 1997]. The secondary structural elements were predicted using PSIPRED[Robert and Gouet, 2014]. The 3-D model structure of VDE enzyme (Chlamydomonas CVDE) was constructed using the multiple identity template module of homology modeling tool MODELLER v9.17[Buchan et al., 2013]. The best structure was selected according to the lowest discrete optimized protein energy (DOPE) score criteria and the side chain was further optimized using What if tool[Eswar et al., 2006].

The 3-D structure of VDE was retrieved from the protein data bank (PDB) with PDB ID - 3CQR. Both the refined CVDE and VDE structures were subjected to energy minimization using GROMACS [40]. The final energy-optimized model was validated by model validation server SAVeS (http://nihserver.mbi.ucla.edu/SAVES/). Further, for the model quality assessment and validation, PROCHECK [Vriend, 1990] and ERRAT [Abraham et al., 2015; Laskowski et al., 1993] were used. Energy profile characterization was performed using ProSA [Colovos and Yeates, 1993] and ProQ [Wiederstein and Sippl, 2007]. Analysis of bond lengths and bond angles was executed by MolProbity [Wallner and Elofsson, 2003].

\section{2. $\mathbf{p K}_{\mathrm{a}}$ Calculation}

Multi-conformer continuum electrostatics (MCCE) v2.4 [Davis et al., 2007] and DELPHI v.4 [Song et al., 2009] were used to calculate $\mathrm{pKa}$ of residues in both VDE and CVDE at $\mathrm{pH}$ 7. Further, $\mathrm{pK}_{\mathrm{a}}$ was calculated by PDB2PQR [Rocchia et al., 2002] server for comparison and a better accuracy. The PDB2PQR tool is integrated with several force field options, which was primarily 
designed for Adaptive Poisson-Boltzmann Solver (APBS) calculations. In the calculation process, structures were refined and reconstructed by fixing the missing atoms and several parameters through PDB2PQR like missing hydrogens, assigning atomic partial charges and radii from a specific force field. In the present pKa calculations, CHARMM force field was used [Dolinsky et al., 2007].

\subsection{Quantum Calculation}

Quantum simulation is needed to predict the correct geometry of small molecules like violaxanthin and ascorbic acid substrates in order to provide an accurate description of their binding mode and strength in the active sites of proteins. The Density functional theory (DFT) is presently the most successful approach to compute the electronic-structure properties [Patel et al., 2004]. Popular quantum mechanical descriptors such as the highest occupied molecular orbital (HOMO) and the lowest unoccupied molecular orbital (LUMO) play a major role in governing many chemical reactions[Arguelho et al., 2010; Singh et al., 2011]. Electronic descriptors that correlate to the physicochemical properties of molecules are energies of FMOs, band gap, total energy, dipole moment, ionization potential (IP), electron affinity (EA), electro negativity $(\chi)$, chemical potential $(\mu)$, global chemical hardness $(\eta)$, global chemical softness $(\sigma)$, electrophilicity index $(\omega)$, nucleophilicity $(\mathrm{N})$, and hydrogen bond strength. Geometry optimization was carried out using Gaussian 16 at the level of DFT/B3LYP/6-31+G(d, p) followed by calculations of these DFTbased descriptors by post-processing of Gaussian output files of both the substrates (violaxanthin and ascorbic acid) with a home-built script[Eroğlu et al., 2007]. In assessment of charge densities on atoms in molecules and the optimized geometry, the hybrid DFT functional B3LYP is known to be fairly accurate[Kumari et al., 2014; Remko and von der Lieth, 2006]. Starting with the formatted check point files generated in geometry optimization, the charge density over meshes was calculated by CUBEGEN program to determine the molecular electrostatic potential (MEP) for both the substrates. GaussView [Dennington et al., 2007; Lee et al., 1988] program was used to visualize the molecular structure and partly to do analysis.

\subsection{Molecular Docking}

In view of comparing between the roles of VDE and CVDE in the xanthophyll cycle, understanding the details of binding of ascorbic acid and violaxanthin with CVDE and VDE is 
imperative. According to previous studies of the xanthophyll cycle, VDE is catalyzed by violaxanthin as well as ascorbic acid. This is conceptualized for CVDE in the current work to explore the binding mechanism. The structures of ascorbic acid and violaxanthin were obtained from Zinc database[Frisch et al., 2009] followed by geometry optimization by DFT. Using their optimized geometries, molecular docking was carried out with the protein enzymes by Auto Dock v4.2.3 program [Irwin and Shoichet, 2005]. A web based server 3-D Ligand site was used to determine grid values after identification of pocket binding residues [Morris et al., 2009]. Total 4 docking simulations were performed for two proteins with each of the two substrates. For accuracy, the torsional degrees of freedom, atomic partial charges, non-polar hydrogens and rotatable bonds of the substrates were taken into consideration while docking. A $65 \times 65 \times 65$ grid box with spacing between points of $0.375 \AA$ was used. Docking scores of substrate-protein complexes were analyzed by the Lamarckian Genetic Algorithm (LGA). The docked conformations were clustered with a $2 \AA$ cut-off root mean square deviation (RMSD) and subsequently ranked according to their binding energy scores. PyMol was used for visualization and 2-D interaction plots were made by BIOVIA Discovery Studio Visualizer v4.5 (BIOVIA DSV) for the non-bonded interactions including hydrogen bond, van der Walls, $\pi-\pi$ and other electrostatic interactions between the substrates and proteins. All of the four substrate-protein complexes were subjected to Molecular Dynamics thereafter for better understanding of interactions and stability of complex formation.

\subsection{Molecular Dynamics (MD) Simulation of Complexes}

GROMACS v2018 was used for carrying out molecular dynamics simulations to judge the stability, compact form, and molecular interactions of the complexes under dynamical situations at room temperature. The topology of the substrates was prepared using AnteChamber Python Parser interfaceE (ACPYPE) and PRODRG server [Wass et al., 2010]. The complexes were inserted in a cubical box containing extended simple point charge (SPC/E) water molecules and the GROMOS96 53a6 force field was used for the protein enzymes[da Silva and Vranken, 2012]. Counter ions $\left(\mathrm{Na}^{+} / \mathrm{Cl}^{-}\right)$corresponding to the physiological strength of $0.15 \mathrm{M}$ were added to neutralize each simulation system. To avoid steric conflicts between atoms and high energy interactions, each electro-neutralized system was subjected to steepest descent energy minimization. After energy minimization, each system was treated with position restrained 
simulations in NVT and NPT ensemble. Each simulation consists of equilibration under NVT (canonical) ensemble for $1 \mathrm{~ns}$ at $300 \mathrm{~K}$ and subsequent $20 \mathrm{~ns}$ simulation under NPT ensemble during which the positions of the backbone of proteins were kept restrained. To constrain the covalent bonds, the Linear Constraint Solver (LINCS) algorithm was applied and electrostatic interactions were calculated using the particle mesh Ewald (PME) method. The cut-off radii for the coulomb and van der Waals interactions were fixed at $10.0 \AA$ and $14.0 \AA$, respectively. The resultant MD trajectories saved at the interval of 100 ps were analyzed using the built-in modules of GROMACS and visual molecular dynamics (VMD 1.9.1)[Schuler et al., 2001]. 2-D plots depicting the intrinsic dynamical stabilities captured by the root mean square deviation (RMSD), root mean square fluctuation (RMSF), and radius of gyration ( $\mathrm{Rg}$ ) of the complexes were generated by Grace 5.1.23 program.

\subsection{Principal Component Analysis (PCA)}

Essential dynamics (ED) or principal component analysis (PCA) is an efficient statistical method that is applied to reduce the number of dimensions needed to describe protein molecular dynamics through a decomposition process [Humphrey et al., 1996]. PCA is a linear transformation that extracts the most important data using a covariance matrix or a correlation matrix (normalized PCA) modeled from atomic coordinates that describe the accessible degrees of freedom (DOF) of a protein, such as the cartesian coordinates that define atomic displacements or movements in each conformation in a trajectory. This covariance matrix is diagonalized to extract a set of eigenvectors and eigenvalues that reflect the correlated concert motion of the molecule [Amadei et al., 1993; Zhou et al., 2006]. The Gromacs module tool called g_covar was used to yield the eigenvalues and eigenvectors by calculating and diagonalizing the covariance matrix, whereas the g_anaeig tool was used to analyze and plot the eigenvectors[Swain et al., 2018].

\subsection{Binding Free Energy Calculation}

Molecular Mechanics/Poisson-Boltzmann Surface Area (MM/PBSA) is widely used for free energy calculation from MD trajectory. This method has been adopted to determine the binding affinity of substrate-protein complexes in many previous works in the post-molecular dynamics analysis[Van Aalten et al., 1995; Wang et al., 2018]. The binding free energy $\left(\Delta \mathrm{G}_{\text {bind }}\right)$ in a solvent medium was calculated as follows: 


\section{$\Delta \mathbf{G}_{\text {bind }}=\mathbf{G}_{\text {complex }}-\left(\mathbf{G}_{\text {protein }}+\mathbf{G}_{\text {substrate }}\right)$}

Where $G_{\text {complex }}$ is the total free energy of the substrate-protein complex, $G_{\text {protein }}$ and $G_{\text {substrate }}$ are the total energies of protein and substrate alone in a solvent, respectively. The free energies for each individual $\mathrm{G}_{\text {complex }}, \mathrm{G}_{\text {protein }}$ and $\mathrm{G}_{\text {substrate }}$ were estimated by

\section{$\mathbf{G}_{\mathbf{p}}=\mathbf{E}_{\mathbf{M M}}+\mathbf{G}_{\text {solv }}$}

Where $\mathrm{p}$ can be protein, substrate, or complex. $\mathrm{E}_{\mathrm{MM}}$ is the average molecular mechanics potential energy in vacuum and $\mathrm{G}_{\text {solv }}$ is the solvation free energy. The molecular mechanics potential energy was calculated in vacuum as follows:

\section{$\mathbf{E}_{M M}=\mathbf{E}_{\text {bonded }}+\mathbf{E}_{\text {non-bonded }}=\mathbf{E}_{\text {bonded }}\left(\mathbf{E}_{\text {int }}\right)+\mathbf{E}_{\mathbf{v d w}}+\mathbf{E}_{\text {elec }}$}

Where $\mathrm{E}_{\text {bonded }}$ or $\mathrm{E}_{\text {int }}$ is bonded interaction, which includes all bonded interactions like bond, angle, dihedral and improper interactions, and $\mathrm{E}_{\text {non-bonded }}$ is non-bonded interaction consisting of the sum of both van der Waals $\left(\mathrm{E}_{\mathrm{vdw}}\right)$ and electrostatic $\left(\mathrm{E}_{\mathrm{elec}}\right)$ interactions. Ebonded is always taken as zero. The solvation free energy $\left(\mathrm{G}_{\text {solv }}\right)$ was estimated as the sum of electrostatic solvation free energy $\left(\mathrm{G}_{\text {polar }}\right)$ and nonpolar solvation free energy $\left(\mathrm{G}_{\text {non-polar }}\right)$

$\mathbf{G}_{\text {solv }}=\mathbf{G}_{\text {polar }}+\mathbf{G}_{\text {non-polar }}$

Where $G_{\text {polar, }}$, the polar solvation energy was determined and computed using the Poisson-

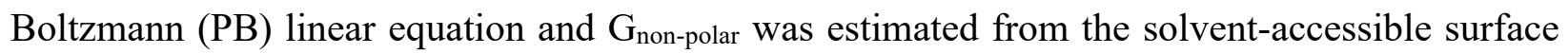
area (SASA) as per the following equation:

\section{$\mathbf{G}_{\text {non-polar }}=\gamma \mathbf{S A S A}+\mathbf{b}$}

Where $\gamma$ (coefficient related to surface tension of the solvent) $=0.02267 \mathrm{~kJ} / \mathrm{mol} / \AA^{2}$ or 0.0054 $\mathrm{kcal} / \mathrm{mol} / \AA^{2}$

$\mathrm{b}=3.849 \mathrm{~kJ} / \mathrm{mol}$ or $0.916 \mathrm{kcal} / \mathrm{mol}$

In this study, the binding free energies for all four complexes were calculated based on 1000 snapshots taken at an equal interval of time from $20 \mathrm{~ns}$ MD simulations. The per-residue energy contribution was also computed to understand the contribution of individual amino acids to the total binding energy. 


\section{Results and Discussion}

\subsection{Structural Overview and 3D Model Validation of CVDE}

The conserved domain analysis shows that CVDE has a large domain of FAD binding-3 super family (PSSM ID-332373). This domain is involved in FAD binding in a number of enzymes. There are evidences that CVDE as hydroxybenzoate hydroxylase, a well-known member of this superfamily, exhibits catalytic behavior. Template identification through BLASTp search reveals a template, i.e. the crystal structure of 3-hydroxybenzoate hydroxylase (PDB ID- 2DHK) with a sequence identity of $73 \%$ based on the target - template alignment (see Figure S1 of the Supporting Information). Employing Modeller v9.04, among the raw 3-D models developed, the model with the lowest DOPE score and RMSD value (on structural superposition on corresponding $\mathrm{C} \alpha$ atom pairs) with regard to the template structure is selected for further refinement. Loops were refined through Galaxy Loop refinement protocol, whereas the side chain improvement was performed using What - If. The refined model of CVDE was subjected to stereochemical quality assessment using various protein structure evaluation tools (see Table S1 and Figures S2, S3A-D) such as PROCHEK, Verify 3D, ERRAT, etc., and secondary sequence analysis by PSIPRED server (Figure S2). The accuracy of the model as per PROCHECK, integrated in Ramachandran plot, based on the dihedral (Phi and Psi) angles of amino acid residues is assessed to be 91.2\% residues in the allowed region of the plot having an average $\mathrm{G}$ - factor score of 0.2 (Figure S3C). No residues are in the disallowed region of the Ramachandran plot which indicates quite high quality/accuracy of the model used (Figure S3C). Therefore, all the stereochemical properties of the model are within the acceptable range. The predicted model has a Verify3D score of $88.79 \%$, indicating the quality of the model is reasonably good as before. According to ProSA, the energy profile analysis (Table S1 and Figure S3D gives a Z-score of -4.54; ProQ analysis also indicates extremely good quality of the model based on their MaxSub $(0.389<1.0)$ and LG scores $(3.40<$ 5.0). Bond length and bond angle analyses of the proposed models in MolProbity show none of the residues has bad side chains or main chain conflicts. Summarily, all the data for model validation are presented in Table S1 that contains as well the similar data for VDE (PDB ID3CQR) for comparison. The overall analyses ensure the proposed model of CVDE is of good quality and can be considered for further studies. To assess the structural integrity of the modeled protein, architectural data on the 3-D structure analyzed with different tools are discussed next. 
Figures S3 represents the 3-D model structure and the topology of CVDE in solid-ribbon. The modeled CVDE structure has no identified barrel fold except thirteen $\alpha$ - helices together with twenty-three $\beta$ strands as seen in Figure S3A. The modeled structure consisting of 4 sheets $(1-$ parallel beta sheet, 1 - antiparallel-beta sheet, and 2 beta-alpha units with 61 residues in loop and 32 in helix); 8 beta hairpins, 12 beta bugles, 3-gama turns, 59 beta turns ( 29 h-bonds). Of 849 amino acids (aa) of the entire protein structure, 216 aa contribute to $\alpha$-helices (24.65\%), 292 aa to $\beta$ strands (33.23\%), 5 aa (1.8\%) and the rest 368 aa (42.09\%), respectively, to turns and coils. In VDE (PDB ID-3qcr), the C-terminal pole of the $\alpha / \beta$ barrel is found to be composed of loops with less than 5 aa over a short length. These are different from the loops at $\mathrm{N}$ - terminal pole which usually acts as an active binding site as evident in catalysis and other ligand binding mechanisms. Unlike VDE, CVDE does not have any specific evidence for its binding mechanisms through both the terminals, which urges for a deeper structural investigation to shed light on its catalytic properties.

\subsection{DFT Studies: Electronic Descriptors}

All the electronic descriptors which were obtained from DFT calculations of the substrates are presented in Table S2. Both the sign and magnitude of total energy express the likelihood of molecules[Dennington et al., 2007; Lee et al., 1988]. The total energy of ascorbic acid is -676.59 Hartree while violaxanthin has -1836.34 Hartree. The large negative values suggest that the molecules are energetically at their local minima having preferable configurations. The dipole moment of violaxanthin is 2.52 Debye while ascorbic acid shows the highest value of 4.04 Debye[Garbett and Chaires, 2012]. Elevated dipole moment enhances the hydrogen bond formation, nonbonding interactions, binding affinity and polar nature of a molecule[Lien et al., 1982]. The electron donating and receiving ability of a molecule can be determined by considering HOMO and LUMO energies of that molecule. Higher the energy of HOMO, higher will be its electron donating ability. It has been found that the energies of HOMOs are comparable for both compounds. Similarly, the LUMO orbital energies are also comparable. The LUMO energy level is affected by the presence of electron donating or withdrawing or both groups in the structure and is responsible for biological activity in addition to other factors. Thus, the study of frontier molecular orbitals (HOMO and LUMO) is quite useful. Chemical hardness ( $\eta$ ) and softness (S) of a molecule can be determined from the LUMO - HOMO gap [Ayers et al., 
2006]. A large gap is related to high kinetic stability and low chemical reactivity; a small gap is indicative of low chemical stability, because the addition of electrons to a high-lying LUMO and/ or removal of electrons from a low-lying HOMO is energetically favorable in any potential chemical reaction [Aihara, 1999]. In this study, ascorbic acid has the HOMO-LUMO gap of $0.35 \mathrm{eV}$ while violaxanthin shows slightly a lower energy gap $(0.29 \mathrm{eV})$. The larger chemical potential $(-0.10 \mathrm{eV})$ along with higher chemical softness $(6.84 \mathrm{eV})$ values may contribute to the higher chemical reactivity of violaxanthin than ascorbic acid (Table S2). The higher electronegativity $(0.17 \mathrm{eV})$ and electrophilicity $(0.09 \mathrm{eV})$ of ascorbic acid indicate its high susceptibility to withdraw electrons than violaxanthin.

\subsection{Docking Analysis}

It is well known that VDE binds with ascorbic acid and violaxanthin during the xanthophyll cycle. There are evidences that violaxanthin travels freely in the lipid phase of cell and does not bind with any pigment carrier proteins [2.18]. Therefore, the hunch is to find out whether these substrates can similarly work with CVDE. Figure 1 provides information about interactions present in the bonded complexes. In docking, the best docking pose is selected based on the highest docking score from the cluster analysis. Information about the highest docking scores, binding efficiency, and the number of h-bonds along with other interactional data for binding in each complex are given in Table 1 . The VDE-ascorbic acid complex has binding affinity $-7.4 \mathrm{kcal} / \mathrm{mol}$, with four conventional h-bonds and two carbon-hydrogen bonds. In case of VDE-violaxanthin, there exist two conventional h-bonds. Despite a large number of h-bonds, VDE-ascorbic acid has a lower bindng affinity than VDE-violaxanthin $(-9.14 \mathrm{kcal} / \mathrm{mol})$. The two complexes of CVDE formed with ascorbic acid and violaxanthin have binding affinities $-6.4 \mathrm{kcal} / \mathrm{mol}$ and -9.94 $\mathrm{kcal} / \mathrm{mol}$ with the number of conventional h-bonds being three and five, respectively. Owing to the highest number of residues participating in the van dar Waals interaction, CVDE-violaxanthin has the strongest binding affinity among all. Despite slight variations, post-MD docking analyses also reveal the same trend in binding affinity among the substrate-enzyme complexes.

\subsection{Salt-bridge Analysis}

Salt-bridge plays a pivotal role in the thermo-stability of enzymes aiding to deduce different plausible reaction mechanisms[Charbonneau and Beauregard, 2013]. Table S4 provides details of 
salt-bridges and $\mathrm{pK}_{\mathrm{a}}$ of residues in the enzyme complexes. While the initial configurations of the complexes possess a very few intramolecular salt-bridges, there is a significant increase in number after the MD run. All over the CVDE complexes retain a substantial number of salt-bridges compared to that of VDE. In reference to the initial configurations identified with 3, 4, 13, and 57 salt-bridges, the optimized complexes after MD have 37, 50, 232, and 245 salt-bridges, respectively, for VDE-ascorbic acid, VDE-violaxanthin, CVDE-ascorbic acid, and CVDEviolaxanthin. Moreover, CVDE-violaxanthin and CVDE-ascorbic acid possess namely 103 and 87 new salt-bridges, which are formed at the side chains contributing to the self-stability of the protein[Charbonneau and Beauregard, 2013; Meuzelaar et al., 2016; Pace et al., 2009]. There is no intermolecular salt-bridge seen between the protein enzymes and substrates whatsoever. Table S3 reports salt-bridges which sustain before and after the MD simulation. $\mathrm{pK}_{\mathrm{a}}$ calculations across the residues demonstrate that a lower average $\mathrm{pK}_{\mathrm{a}}$ value is crucial for having a greater number of saltbridges in the complexes (see Table S4). This implies that lower the difference $(\Delta)$ of the average $\mathrm{pK}_{\mathrm{a}}$ between residues with and without a salt-bridge, chances of having more salt-bridges in the complexes are higher and vice-versa.

Taken into account the total number of acidic and basic residues present in VDE and CVDE, which are actually responsible for the salt-bridge formation, the number of salt-bridges formed per residue is larger for the violaxanthin complexes than that of ascorbic acid. Again, this number is larger for CVDE than VDE. As there exists a larger average no. of salt-bridges in CVDE or the violaxanthin complexes, consequently the protein-protein interaction is more there and the scope of interaction with the substrate(s) is less resulting in a weaker bonding between the corresponding enzymes and the substrates, which justifies the similar trend of binding free energies reported in Table 2 .

\subsection{Trajectory Analysis}

From the MD simulations, the intrinsic dynamical stability of each complex was studied by the root-mean-square deviation (RMSD) of the backbone, radius of gyration $\left(\mathrm{R}_{\mathrm{g}}\right)$, solvent accessible surface area (SASA) and root-mean-square fluctuation (RMSF) of the $\mathrm{C}_{\alpha}$ atom as a function of simulation time, which are shown in Figure 2. RMSD measures the difference between the final and initial positions of the backbone of a protein. By means of deviations plotted over the course of simulation, the stability of a protein-ligand complex relative to its preliminary state can be easily 
assessed. A smaller deviation indicates the greater stability of protein-ligand complexes. Of the VDE- and CVDE-ascorbic acid complexes, the RMSD plot of VDE is smooth and nearly flat with an average backbone deviation of $0.45 \AA$ in comparison to $0.63 \AA$ of the latter where fluctuations as well as an increasing trend of RMSD are obvious, see Figure 2(i) A \& A1. Exhibiting quite similar trends between VDE- and CVDE-violaxanthin complexes as of the ascorbic acid complexes, the average backbone deviations are, respectively, $0.41 \AA$ and $0.76 \AA$, which are the highest and the least value of RMSD among all and so does their stability (Figure 2(ii) A \& A1).

The radius of gyration $\left(\mathrm{R}_{\mathrm{g}}\right)$ plots about a common center of mass are shown in $\mathrm{C} \& \mathrm{C} 1$ of Figure 2(i) \& (ii), with the CVDE complexes having larger values than the VDE complexes. In the VDE complexes, the average $\mathrm{R}_{\mathrm{g}}$ values are $1.56 \AA$ and $1.59 \AA$, respectively, for ascorbic acid and violaxanthin, whereas $2.64 \AA$ and $3.76 \AA$ are the same for the CVDE complexes. Hence, in terms of forming a more compact complex, VDE is better than CVDE.

Otherwise, this becomes transparent in the plots of the solvent accessible surface area (SASA) of the proteins, D \& D1 of Figure 2(i) \& (ii), where the complexes of VDE have smaller surface areas than that of CVDE with the average values, respectively: 77.9 and $379 \AA^{2}$ (for violaxanthin); 85.6 and $363 \AA^{2}$ (for ascorbic acid). Towards ligand binding strength and the stability of the complexes, the RMSF plots (B \& B1 of Figure 2(i) \& (ii)) for the $\mathrm{C}_{\alpha}$ atom and the average values are also quite insightful. Compared to VDE, the plots for CVDE contain more noises and larger RMSF values, which indicate relatively a weak binding between CVDE and the substrates. As the average RMSF increases in the order for VDE: VDE-violaxanthin $(0.25 \mathrm{~nm})<$ VDE-ascorbic acid $(0.32$ $\mathrm{nm})$ and in case of CVDE: CVDE-violaxanthin $(0.72 \mathrm{~nm})<$ CVDE-ascorbic acid $(0.87 \mathrm{~nm})$, the binding strength and hence the stability of the protein-substrate complexes should decline in the same sequence. Worthwhile to mention that a few regions including the variable loops show greater fluctuations in their $\mathrm{C}_{\alpha}$ atoms in all the systems indicating more flexibility of these residues. In both the complexes of CVDE, a significant fluctuation occurs at aa residues 50 to 60 and 90 to 100 , with the highest peak located at the $100^{\text {th }}$ aa residue. Some extra fluctuations after $860^{\text {th }}$ residue also contribute to the RMSF of CVDE-violaxanthin.

\subsection{Intermolecular H-bond Analysis}


H-bond is one of the major driving interactions contributing to the stability of the complexes. Using gmx hbond, h-bonds formed over the simulation period were analyzed in all the complexes and are presented in Figure 3. Compared to the ascorbic acid complexes, the violaxanthin complexes manifest a consistent pattern of h-bond formation. Among all, VDE-ascorbic acid has by far the largest number of h-bonds (average: 3.91 ) and the corresponding CVDE complex has the lowest number (average: 1.33). On the contrary, the violaxanthin complexes of VDE and CVDE have a consistent pattern over time with the average numbers of h-bonds being comparable, 1.95 and 2.21, respectively. Considering the overall aspect of dynamical variations of the intermolecular h-bond and binding affinity, it is immediately evident that CVDE and VDE form more stable complexes with violaxanthin than with ascorbic acid.

\subsection{Secondary Structure Analysis}

With gmx DSSP tool of GROMACS, secondary structure analysis of all the complexes was performed. This study helps us to examine the secondary structure of proteins, which contains helices, beta-sheets and loops, and associated internal motions over time (see Figure 4). In both the complexes of CVDE, formation of coil is predominant, around 50\% residues which are part of this don't play any role in structure formation. Around 20\% residues not forming any h-bonds are present, which also don't help in structure build-up. Residues capable of exhibiting h-bond interactions i.e. alpha -helices account for another 20\%. In passing, the presence of 5-helices and turns gives rise to a large number of h-bond formations in VDE-violaxanthin compared to VDEascorbic acid. Due to more parallel and antiparallel backbone h-bonds present in the beta-sheet, CVDE-violaxanthin has the more stable secondary structure than CVDE-ascorbic acid. While beta-sheets present at large proportion in both the complexes of VDE, most of the residues participate in structure formation. Interestingly, the maximum number of h-bonds shown by VDEascorbic acid arises from prevailing structural characteristics of different types of motifs helping in secondary structure formation such as alpha-helices, beta-sheet, beta-bridge, and turns. As most of the beta-bridge residues help in beta-sheet formation in CVDE-violaxanthin than CVDEascorbic acid, the latter exhibits inferiority in the formation of alpha-helices due to the lack of rigidity and stability.

\subsection{Binding Free Energy Calculation}


The binding free energy and its various components obtained from MM/PBSA [Kumari et al., 2014; Remko and von der Lieth, 2006] calculations of the CVDE and VDE complexes are listed in Table 5. Free energy calculations indicate that both CVDE and VDE with violaxanthin possess the highest negative binding free energy of $-119.28 \pm 55.87 \mathrm{~kJ} \mathrm{~mol},-177.40 \pm 86.51 \mathrm{~kJ} \mathrm{~mol}$, respectively, as compared to the corresponding ascorbic acid systems. In case of all the enzymes, the polar solvation (ps) energy is positive, against the binding with ascorbic acid and violaxanthin; while the rest such as the van der Waals (vdW), electrostatic (ele), and solvent accessible surface area (sasa) energies are negative favoring binding. Among the favorable interactions, ele and sasa are the least contributor, respectively, for the violaxanthin and ascorbic acid acid complexes. Whereas, the vdW interaction has the largest contribution to binding almost for all. The high negative value of vdW energy suggests that a hydrophobic interaction substantially interplays between VDE and violaxanthin, CVDE and violaxanthin/ascorbic acid. Based on the binding free energy analysis, it can be demonstrated that both CVDE and VDE systems with violaxanthin appear to exhibit the most intrinsic dynamical stability than with ascorbic acid. This is in consistent with the earlier prediction based on the binding energies reported in Table 2. These findings explain as to why VDE and violaxanthin taking part in many catalytic processes reported in the literature. In the experimental work of Meireles et al. ${ }^{31}$, the ascorbate deficiency does not affect the de-epoxidation process in CVDE, suggesting that ascorbate is not required for this process. The lower negative binding energy/free energy of CVDE-ascorbic acid here indeed confirms this experimental finding, undermining the role of ascorbic acid in de-epoxidation for CVDE compared to violaxanthin.

\subsection{PCA}

In PCA, essential dynamics (ED) was employed to represent the directions of principal motions by a set of eigenvectors (EV) called principal or essential modes. Each MD trajectory was projected onto the phase space to yield a spectrum of EVs, which depict the vectorial representation of each single components in motion. Each EV holds an eigenvalue that describes the energetic contribution of each component to the motion (Figures 5A, C, E, and G). It is observed that 75$77 \%$ of the backbone motion is covered by the first $20 \mathrm{EVs}$ where an exponentially decaying curve of eigenvalues is obtained against the EVs (Figure 5). The differential scattering of atoms in contour-like plots specifies the occurrence of conformational changes in the complexes in 
agreement with other MD analyses. Figures 5B, D, F, and $\mathrm{H}$ reveal a higher subspace dimension in both the complexes of CVDE. In contrast to CVDE, the VDE systems cover the least subspace and show the least variations while the first two PCs were taken into consideration. In the 2-D projection plots of trajectories, the CVDE systems show higher trace values of the covariance matrix than the VDE systems. In the overall PCA analysis, we conclude that the CVDE systems have more flexibility than the VDE systems in which the VDE-violaxanthin complex shows the least conformational changes due to decreasing collective motions than other systems. This result is in congruent with the stability of the VDE-violaxanthin complex in the xanthophyll cycle during de-epoxidation chemical process. Emphatically, the PCA and RMSF analyses elicit the same characteristics about structural fluctuations of the protein-substrate complexes.

\section{Conclusion}

Violaxanthin de-epoxidase (VDE) plays a pivotal role in xanthophyll cycle and keeps photosynthesis process intact by protecting from reactive oxygen species generated during the process. Very recently, a variant of VDE (called Chlamydomonas CVDE) present in algae is discovered, which functionally resembles with VDE enzyme but was not explored. To understand whether there exist any structural and functional similarities, a comparative study is performed between VDE and CVDE shedding light on the primary steps of the xanthophyll (photosynthesis) cycle. Hence, phylogenetic profile, Bioinformatics analyses for structure determination and modeling, molecular dynamics studies (to check binding stability, fluctuations, energetics, etc.) of the enzyme complexes with the same substrates are performed. It is found that CVDE is an ancestor of VDE. Like VDE, CVDE has a catalytic domain even larger than that of VDE, thus can exhibit catalytic properties. From the essential dynamics (ED), RMSD, $\mathrm{R}_{\mathrm{g}}$, and intermolecular h-bonds, CVDE displays higher conformational changes compared to VDE, which may attribute to its larger structure having more residues than VDE. Evaluation and decomposition of binding free energies by MM/PBSA demonstrate that the van der Waals and electrostatic interactions majorly contribute to binding with the substrates for both enzymes. Along with the binding energies, the free energy analysis enlightens that both CVDE and VDE have equal affinity to violaxanthin, while VDE binds to ascorbic acid stronger than CVDE. We found out that the experimental result of Meireles et al. is in congruent with our study, indicating that the deepoxidation process in CVDE requires different reductants than ascorbic acid. Overall, the study 
discerns that CVDE might have similar roles as VDE in photosynthesis/photoprotection in the presence of violaxanthin and trivial roles with ascorbic acid. Taken everything into consideration, this work presents new insights into structural details and binding mechanisms of CVDE that were unknown till date as compared to widely studied VDE, which may incite more theoretical and experimental studies in this direction as far as photosynthesis or the xanthophyll cycle is concerned.

\section{Conflict of interest}

The authors declare no conflict of interest and all the authors have approved this article for publication.

\section{Acknowledgements}

The authors acknowledge IISER Berhampur for computational support. P.S.S.G also sincerely acknowledges IISER Berhampur for providing him the Institute Postdoc Fellowship to carry out this work.

\section{REFERENCE}

Abraham MJ, Murtola T, Schulz R, Páll S, Smith JC, Hess B, Lindahl E. 2015. GROMACS: High performance molecular simulations through multi-level parallelism from laptops to supercomputers. SoftwareX 1:1925.

Aihara J-i. 1999. Reduced HOMO- LUMO gap as an index of kinetic stability for polycyclic aromatic hydrocarbons. The Journal of Physical Chemistry A 103:7487-7495.

Altschul SF, Madden TL, Schäffer AA, Zhang J, Zhang Z, Miller W, Lipman DJ. 1997. Gapped BLAST and PSIBLAST: a new generation of protein database search programs. Nucleic acids research 25:3389-3402.

Amadei A, Linssen AB, Berendsen HJ. 1993. Essential dynamics of proteins. Proteins: Structure, Function, and Bioinformatics 17:412-425.

Anwaruzzaman M, Chin BL, Li X-P, Lohr M, Martinez DA, Niyogi KK. 2004. Genomic analysis of mutants affecting xanthophyll biosynthesis and regulation of photosynthetic light harvesting in Chlamydomonas reinhardtii. Photosynthesis Research 82:265-276. 
Arguelho MLP, Alves JdPH, Stradiotto NR, Lacerda Júnior V, Pires JM, Beatriz A. 2010. Electrochemical and theoretical evaluation of the interaction between dna and amodiaquine: evidence of the guanine adduct formation. Química Nova 33:1291-1296.

Arnoux P, Morosinotto T, Saga G, Bassi R, Pignol D. 2009. A structural basis for the pH-dependent xanthophyll cycle in Arabidopsis thaliana. The Plant Cell 21:2036-2044.

Ayers PW, Parr RG, Pearson RG. 2006. Elucidating the hard/soft acid/base principle: a perspective based on half-reactions. The Journal of chemical physics 124:194107.

Baroli I, Do AD, Yamane T, Niyogi KK. 2003. Zeaxanthin accumulation in the absence of a functional xanthophyll cycle protects Chlamydomonas reinhardtii from photooxidative stress. The Plant Cell 15:9921008.

Bradbury LM, Shumskaya M, Tzfadia O, Wu S-B, Kennelly EJ, Wurtzel ET. 2012. Lycopene cyclase paralog CruP protects against reactive oxygen species in oxygenic photosynthetic organisms. Proceedings of the National Academy of Sciences 109:E1888-E1897.

Bratt CE, Arvidsson P-O, Carlsson M, Åkerlund H-E. 1995. Regulation of violaxanthin de-epoxidase activity by $\mathrm{pH}$ and ascorbate concentration. Photosynthesis Research 45:169-175.

Briantais J-M, Vernotte C, Picaud M, Krause G. 1979. A quantitative study of the slow decline of chlorophyll a fluorescence in isolated chloroplasts. Biochimica et Biophysica Acta (BBA)-Bioenergetics 548:128-138.

Britton G. 1976. Later reactions of carotenoid biosynthesiseditor^editors. Carotenoids-4. Elsevier, p 223236.

Buchan DW, Minneci F, Nugent TC, Bryson K, Jones DT. 2013. Scalable web services for the PSIPRED Protein Analysis Workbench. Nucleic acids research 41:W349-W357.

Bugos RC, Hieber AD, Yamamoto HY. 1998. Xanthophyll cycle enzymes are members of the lipocalin family, the first identified from plants. Journal of biological chemistry 273:15321-15324.

Bugos RC, Yamamoto HY. 1996. Molecular cloning of violaxanthin de-epoxidase from romaine lettuce and expression in Escherichia coli. Proceedings of the national academy of sciences 93:6320-6325.

Bungard RA, Ruban AV, Hibberd JM, Press MC, Horton P, Scholes JD. 1999. Unusual carotenoid composition and a new type of xanthophyll cycle in plants. Proceedings of the National Academy of Sciences 96:1135-1139.

Casper-Lindley C, Björkman O. 1998. Fluorescence quenching in four unicellular algae with different lightharvesting and xanthophyll-cycle pigments. Photosynthesis research 56:277-289.

Charbonneau DM, Beauregard M. 2013. Role of key salt bridges in thermostability of G.

thermodenitrificans EstGtA2: distinctive patterns within the new bacterial lipolytic enzyme family XV. PLoS One 8:e76675.

Coesel S, Oborník M, Varela J, Falciatore A, Bowler C. 2008. Evolutionary origins and functions of the carotenoid biosynthetic pathway in marine diatoms. PloS one 3:e2896. 
Colovos C, Yeates TO. 1993. Verification of protein structures: patterns of nonbonded atomic interactions. Protein science 2:1511-1519.

da Silva AWS, Vranken WF. 2012. ACPYPE-Antechamber python parser interface. BMC research notes $5: 367$.

Davis IW, Leaver-Fay A, Chen VB, Block JN, Kapral GJ, Wang X, Murray LW, Arendall III WB, Snoeyink J, Richardson JS. 2007. MolProbity: all-atom contacts and structure validation for proteins and nucleic acids. Nucleic acids research 35:W375-W383.

Dennington R, Keith T, Millam J. 2007. GaussView, Version 4.1. 2. Semichem Inc., Shawnee Mission, KS. Dolinsky TJ, Czodrowski P, Li H, Nielsen JE, Jensen JH, Klebe G, Baker NA. 2007. PDB2PQR: expanding and upgrading automated preparation of biomolecular structures for molecular simulations. Nucleic acids research 35:W522-W525.

Eroğlu E, Türkmen H, Güler S, Palaz S, Oltulu O. 2007. A DFT-Based QSARs study of Acetazolamide/Sulfanilamide derivatives with carbonic anhydrase (CA-II) isozyme inhibitory activity. International journal of molecular sciences 8:145-155.

Eswar N, Webb B, Marti - Renom MA, Madhusudhan M, Eramian D, Shen My, Pieper U, Sali A. 2006. Comparative protein structure modeling using Modeller. Current protocols in bioinformatics 15:5.6. 1-5.6. 30.

Foyer $\mathrm{CH}$. 2018. Reactive oxygen species, oxidative signaling and the regulation of photosynthesis. Environmental and experimental botany 154:134-142.

Frisch M, Trucks G, Schlegel H, Scuseria G, Robb M, Cheeseman J, Scalmani G, Barone V, Mennucci B, Petersson G. 2009. Gaussian, Inc., Wallingford CT. Gaussian 03, Revision E 1.

Garbett NC, Chaires JB. 2012. Thermodynamic studies for drug design and screening. Expert opinion on drug discovery 7:299-314.

Hager A, Holocher K. 1994. Localization of the xanthophyll-cycle enzyme violaxanthin de-epoxidase within the thylakoid lumen and abolition of its mobility by a (light-dependent) pH decrease. Planta 192:581-589. Hieber AD, Bugos RC, Yamamoto HY. 2000. Plant lipocalins: violaxanthin de-epoxidase and zeaxanthin epoxidase. Biochimica et Biophysica Acta (BBA)-Protein Structure and Molecular Enzymology 1482:84-91. Hieber DA, Bugos RC, Verhoeven AS, Yamamoto HY. 2002. Overexpression of violaxanthin de-epoxidase: properties of C-terminal deletions on activity and pH-dependent lipid binding. Planta 214:476-483.

Humphrey W, Dalke A, Schulten K. 1996. VMD: visual molecular dynamics. Journal of molecular graphics 14:33-38.

Irwin JJ, Shoichet BK. 2005. ZINC- a free database of commercially available compounds for virtual screening. Journal of chemical information and modeling 45:177-182.

Külheim C, Ågren J, Jansson S. 2002. Rapid regulation of light harvesting and plant fitness in the field. Science 297:91-93.

Kumari R, Kumar R, Consortium OSDD, Lynn A. 2014. g_mmpbsa A GROMACS tool for high-throughput MM-PBSA calculations. Journal of chemical information and modeling 54:1951-1962.

Laskowski RA, MacArthur MW, Moss DS, Thornton JM. 1993. PROCHECK: a program to check the stereochemical quality of protein structures. Journal of applied crystallography 26:283-291.

Lee C, Yang W, Parr RG. 1988. Development of the Colle-Salvetti correlation-energy formula into a functional of the electron density. Physical review B 37:785. 
Li Z, Peers G, Dent RM, Bai Y, Yang SY, Apel W, Leonelli L, Niyogi KK. 2016. Evolution of an atypical deepoxidase for photoprotection in the green lineage. Nature plants 2:16140.

Lien EJ, Guo Z-R, Li R-L, Su C-T. 1982. Use of dipole moment as a parameter in drug-receptor interaction and quantitative structure-activity relationship studies. Journal of pharmaceutical sciences 71:641-655. Lunch CK, LaFountain AM, Thomas S, Frank HA, Lewis LA, Cardon ZG. 2013. The xanthophyll cycle and NPQ in diverse desert and aquatic green algae. Photosynthesis research 115:139-151.

Marchler-Bauer A, Lu S, Anderson JB, Chitsaz F, Derbyshire MK, DeWeese-Scott C, Fong JH, Geer LY, Geer RC, Gonzales NR. 2010. CDD: a Conserved Domain Database for the functional annotation of proteins. Nucleic acids research 39:D225-D229.

Merchant SS, Prochnik SE, Vallon O, Harris EH, Karpowicz SJ, Witman GB, Terry A, Salamov A, Fritz-Laylin LK, Maréchal-Drouard L. 2007. The Chlamydomonas genome reveals the evolution of key animal and plant functions. Science 318:245-250.

Meuzelaar H, Vreede J, Woutersen S. 2016. Influence of Glu/Arg, Asp/Arg, and Glu/Lys Salt Bridges on $\alpha$ Helical Stability and Folding Kinetics. Biophysical journal 110:2328-2341.

Morosinotto T, Baronio R, Bassi R. 2002. Dynamics of chromophore binding to Lhc proteins in vivo and in vitro during operation of the xanthophyll cycle. Journal of Biological Chemistry 277:36913-36920.

Morris GM, Huey R, Lindstrom W, Sanner MF, Belew RK, Goodsell DS, Olson AJ. 2009. AutoDock4 and AutoDockTools4: Automated docking with selective receptor flexibility. Journal of computational chemistry 30:2785-2791.

Müller P, Li X-P, Niyogi KK. 2001. Non-photochemical quenching. A response to excess light energy. Plant physiology 125:1558-1566.

Niyogi KK, Björkman O, Grossman AR. 1997. The roles of specific xanthophylls in photoprotection. Proceedings of the National Academy of Sciences 94:14162-14167.

Niyogi KK, Grossman AR, Björkman O. 1998. Arabidopsis mutants define a central role for the xanthophyll cycle in the regulation of photosynthetic energy conversion. The plant cell 10:1121-1134.

Niyogi KK, Truong TB. 2013. Evolution of flexible non-photochemical quenching mechanisms that regulate light harvesting in oxygenic photosynthesis. Current opinion in plant biology 16:307-314.

Pace CN, Grimsley GR, Scholtz JM. 2009. Protein ionizable groups: pK values and their contribution to protein stability and solubility. Journal of Biological Chemistry 284:13285-13289.

Patel S, Mackerell Jr AD, Brooks III CL. 2004. CHARMM fluctuating charge force field for proteins: II protein/solvent properties from molecular dynamics simulations using a nonadditive electrostatic model. Journal of computational chemistry 25:1504-1514.

Quaas T, Berteotti S, Ballottari M, Flieger K, Bassi R, Wilhelm C, Goss R. 2015. Non-photochemical quenching and xanthophyll cycle activities in six green algal species suggest mechanistic differences in the process of excess energy dissipation. Journal of plant physiology 172:92-103. 
Remko M, von der Lieth C-W. 2006. Gas-phase and solution conformations of the $\alpha$-l-iduronic acid structural unit of heparin. Journal of chemical information and modeling 46:1194-1200.

Robert X, Gouet P. 2014. Deciphering key features in protein structures with the new ENDscript server. Nucleic acids research 42:W320-W324.

Rocchia W, Sridharan S, Nicholls A, Alexov E, Chiabrera A, Honig B. 2002. Rapid grid - based construction of the molecular surface and the use of induced surface charge to calculate reaction field energies: Applications to the molecular systems and geometric objects. Journal of computational chemistry 23:128137.

Ruban AV, Johnson MP, Duffy CD. 2012. The photoprotective molecular switch in the photosystem II antenna. Biochimica et Biophysica Acta (BBA)-Bioenergetics 1817:167-181.

Schuler LD, Daura X, Van Gunsteren WF. 2001. An improved GROMOS96 force field for aliphatic hydrocarbons in the condensed phase. Journal of Computational Chemistry 22:1205-1218.

Siefermann-Harms D. 1985. Carotenoids in photosynthesis. I. Location in photosynthetic membranes and light-harvesting function. Biochimica et Biophysica Acta (BBA)-Reviews on Bioenergetics 811:325-355.

Singh R, Suresh K, Prabhu D. 2011. DFT based study of interaction between frontier orbitals of transition metal halides and thioamides. Int J of ChemTech Res 3:1571-1579.

Song Y, Mao J, Gunner M. 2009. MCCE2: improving protein pKa calculations with extensive side chain rotamer sampling. Journal of computational chemistry 30:2231-2247.

Stransky H, Hager A. 1970. The carotenoid pattern and the occurrence of the light-induced xanthophyll cycle in various classes of algae. VI. Chemosystematic study. Archiv fur Mikrobiologie 73:315.

Swain SS, Paidesetty SK, Dehury B, Sahoo J, Vedithi SC, Mahapatra N, Hussain T, Padhy RN. 2018. Molecular docking and simulation study for synthesis of alternative dapsone derivative as a newer antileprosy drug in multidrug therapy. Journal of cellular biochemistry 119:9838-9852.

Van Aalten D, Findlay J, Amadei A, Berendsen H. 1995. Essential dynamics of the cellular retinol-binding protein evidence for ligand-induced conformational changes. Protein Engineering, Design and Selection 8:1129-1135.

Vriend G. 1990. WHAT IF: a molecular modeling and drug design program. Journal of molecular graphics 8:52-56.

Wallner B, Elofsson A. 2003. Can correct protein models be identified? Protein science 12:1073-1086.

Wang C, Greene DA, Xiao L, Qi R, Luo R. 2018. Recent developments and applications of the MMPBSA method. Frontiers in molecular biosciences 4:87.

Wass MN, Kelley LA, Sternberg MJ. 2010. 3DLigandSite: predicting ligand-binding sites using similar structures. Nucleic acids research 38:W469-W473.

Wiederstein M, Sippl MJ. 2007. ProSA-web: interactive web service for the recognition of errors in threedimensional structures of proteins. Nucleic acids research 35:W407-W410.

Yokthongwattana K, Melis A. 2006. Photoprotection, Photoinhibition, Gene Regulation, and Environment, eds Demmig-Adams B, Adams W, Mattoo Aeditor^editors: Springer, Dordrecht, The Netherlands.

Zhou X, Chou J, Wong ST. 2006. Protein structure similarity from principle component correlation analysis. BMC bioinformatics 7:40. 


\section{TABLES}

Table 1: Details about docking scores (binding energy) and interacting key residues of ascorbic acid and violaxanthin with the target enzymes VDE and CVDE.

\begin{tabular}{|c|c|c|c|c|c|c|}
\hline Enzymes & Ligands & 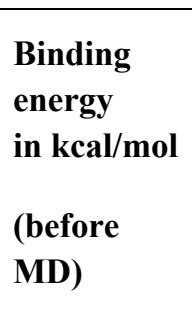 & $\begin{array}{l}\text { Interactin } \\
\text { g key } \\
\text { residues }\end{array}$ & Types of bonding & $\begin{array}{l}\text { Distance } \\
(\AA)\end{array}$ & $\begin{array}{l}\text { Binding } \\
\text { energy } \\
\text { in } \\
\text { kcal/mol } \\
\text { (after } \\
\text { MD) }\end{array}$ \\
\hline \multirow{11}{*}{ CVDE } & \multirow{5}{*}{$\begin{array}{l}\text { Ascorbic } \\
\text { acid }\end{array}$} & \multirow{5}{*}{-6.40} & Ser137 & Conventional hydrogen & 4.85 & \multirow{5}{*}{-5.90} \\
\hline & & & Ala 397 & Conventional hydrogen & 4.54 & \\
\hline & & & Asp146 & Conventional hydrogen & 4.87 & \\
\hline & & & Ser140 & Conventional hydrogen & 3.24 & \\
\hline & & & Thr138 & Conventional hydrogen & 3.42 & \\
\hline & \multirow{6}{*}{ Violaxanthin } & \multirow{6}{*}{-9.94} & Ser462 & Conventional hydrogen & 4.37 & \multirow{6}{*}{-9.60} \\
\hline & & & Ala 529 & Pi-alkyl/alkyl & 4.24 & \\
\hline & & & Leu 491 & Pi-alkyl/alkyl & 4.34 & \\
\hline & & & Ala 463 & Pi-alkyl/alkyl & 4.55 & \\
\hline & & & Ala 593 & Pi-alkyl/alkyl & 4.23 & \\
\hline & & & Glu 493 & Conventional hydrogen & 4.41 & \\
\hline \multirow{5}{*}{ VDE } & \multirow{5}{*}{$\begin{array}{l}\text { Ascorbic } \\
\text { acid }\end{array}$} & \multirow{5}{*}{-7.40} & $A \ln 119$ & Carbon hydrogen & 5.18 & \multirow{5}{*}{-7.10} \\
\hline & & & Pro111 & Pi-alkyl/alkyl & 4.44 & \\
\hline & & & Tyr198 & Conventional hydrogen & 5.85 & \\
\hline & & & Asn110 & Conventional hydrogen & 4.00 & \\
\hline & & & Thr112 & Conventional hydrogen & 2.48 & \\
\hline
\end{tabular}




\begin{tabular}{|c|c|c|c|c|c|}
\hline \multirow{16}{*}{ Violaxanthin } & \multirow{16}{*}{-9.14} & Thr112 & Conventional hydrogen & 2.29 & \multirow{16}{*}{-9.40} \\
\hline & & Ala115 & Pi-alkyl/alkyl & 4.49 & \\
\hline & & Ile105 & Pi-alkyl/alkyl & 5.48 & \\
\hline & & Asn 110 & Conventional hydrogen & 4.50 & \\
\hline & & Ala211 & Conventional hydrogen & 3.91 & \\
\hline & & Val212 & Pi-alkyl/alkyl & 5.73 & \\
\hline & & Tyr198 & Pi-alkyl/alkyl & 6.48 & \\
\hline & & Cys 118 & Pi-alkyl/alkyl & 4.48 & \\
\hline & & Ile105 & Pi-alkyl/alkyl & 4.30 & \\
\hline & & Ile243 & Pi-alkyl/alkyl & 5.52 & \\
\hline & & Val212 & Pi-alkyl/alkyl & 4.68 & \\
\hline & & Ala115 & Pi-alkyl/alkyl & 4.11 & \\
\hline & & Pro111 & Pi-alkyl/alkyl & 4.31 & \\
\hline & & Ala211 & Conventional hydrogen & 3.57 & \\
\hline & & Leu109 & $\begin{array}{l}\text { Unfavorable donor- } \\
\text { donor }\end{array}$ & 4.40 & \\
\hline & & Asn 110 & Conventional hydrogen & 4.28 & \\
\hline
\end{tabular}

Table 2: Binding free energies of the CVDE and VDE Complexes.

\begin{tabular}{|l|l|l|l|l|}
\hline Energy components $(\mathrm{kJ} / \mathrm{mol})$ & VDE_ASC & VDE_VOL & CVDE_ASC & CVDE_VOL \\
\hline van der Waal energy $\left(\Delta \mathrm{G}_{\mathrm{vdw}}\right)$ & $-75.01 \pm 46.07$ & $-237.34 \pm 124.53$ & $-84.99 \pm 38.84$ & $-170.69 \pm 0.52$ \\
\hline Electrostatic energy $\left(\Delta \mathrm{G}_{\mathrm{ele}}\right)$ & $-92.94 \pm 17.32$ & $-5.05 \pm 6.50$ & $-38.67 \pm 22.55$ & $-4.64 \pm 11.07$ \\
\hline Polar solvation energy $\left(\Delta \mathrm{G}_{\mathrm{ps}}\right)$ & $96.30 \pm 31.04$ & $87.83 \pm 57.88$ & $77.03 \pm 40.19$ & $75.77 \pm 43.99$ \\
\hline SASA energy $\left(\Delta \mathrm{G}_{\text {sasa }}\right)$ & $-17.84 \pm 9.49$ & $-22.85 \pm 11.94$ & $-7.94 \pm 3.30$ & $-19.91 \pm 3.99$ \\
\hline Binding energy $\left(\Delta \mathrm{G}_{\text {binding }}\right)$ & $-89.49 \pm 21.07$ & $-177.40 \pm 86.51$ & $-54.57 \pm 29.15$ & $-119.28 \pm 55.87$ \\
\hline
\end{tabular}


bioRxiv preprint doi: https://doi.org/10.1101/2020.06.07.138495; this version posted June 8, 2020. The copyright holder for this preprint (which was not certified by peer review) is the author/funder, who has granted bioRxiv a license to display the preprint in perpetuity. It is made available under aCC-BY-NC-ND 4.0 International license.

\section{FIGURES}
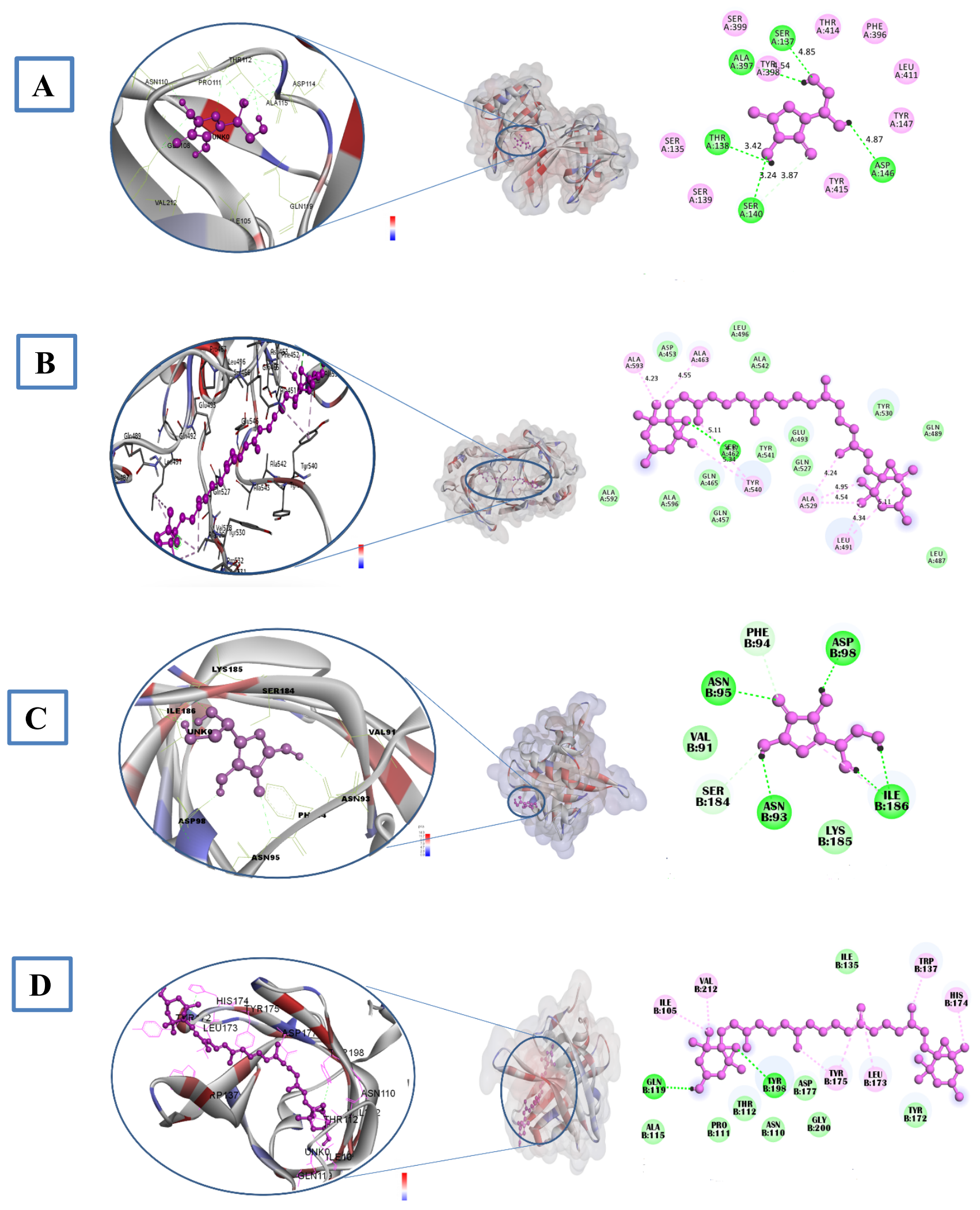


\section{Interactions}

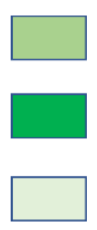

Van der Waals

Unfavorable Donor-Donor

\section{Conventional Hydrogen Bond}

Alkyl

\section{Carbon Hydrogen Bond}

Pi-Alkyl

Figure 1: Interacting key residues and binding modes in (A) CVDE-ascorbic acid, (B) CVDE-violaxanthin, (C) VDEascorbic acid, and (D) VDE-violaxanthin complexes.

(i)
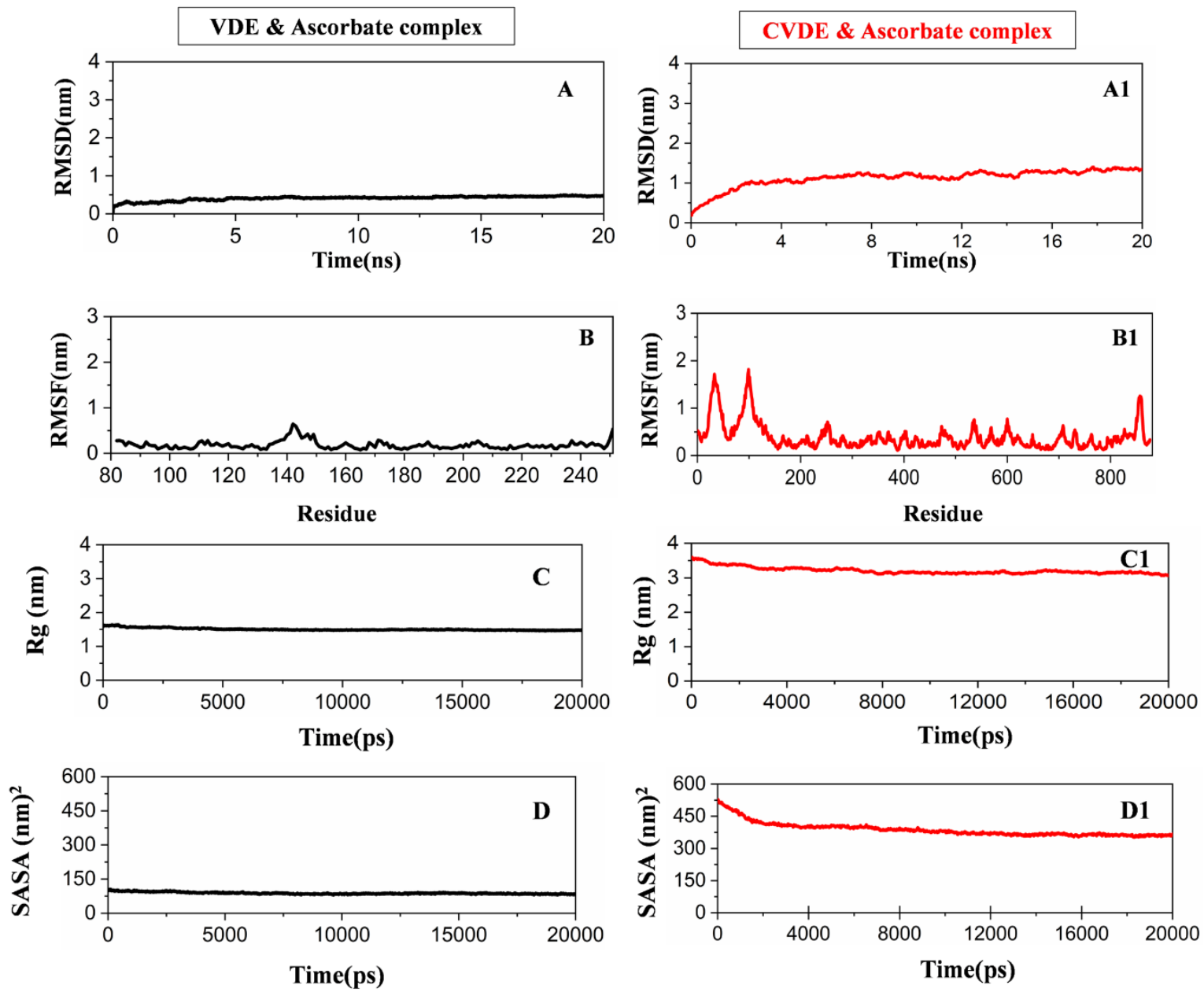
(ii)
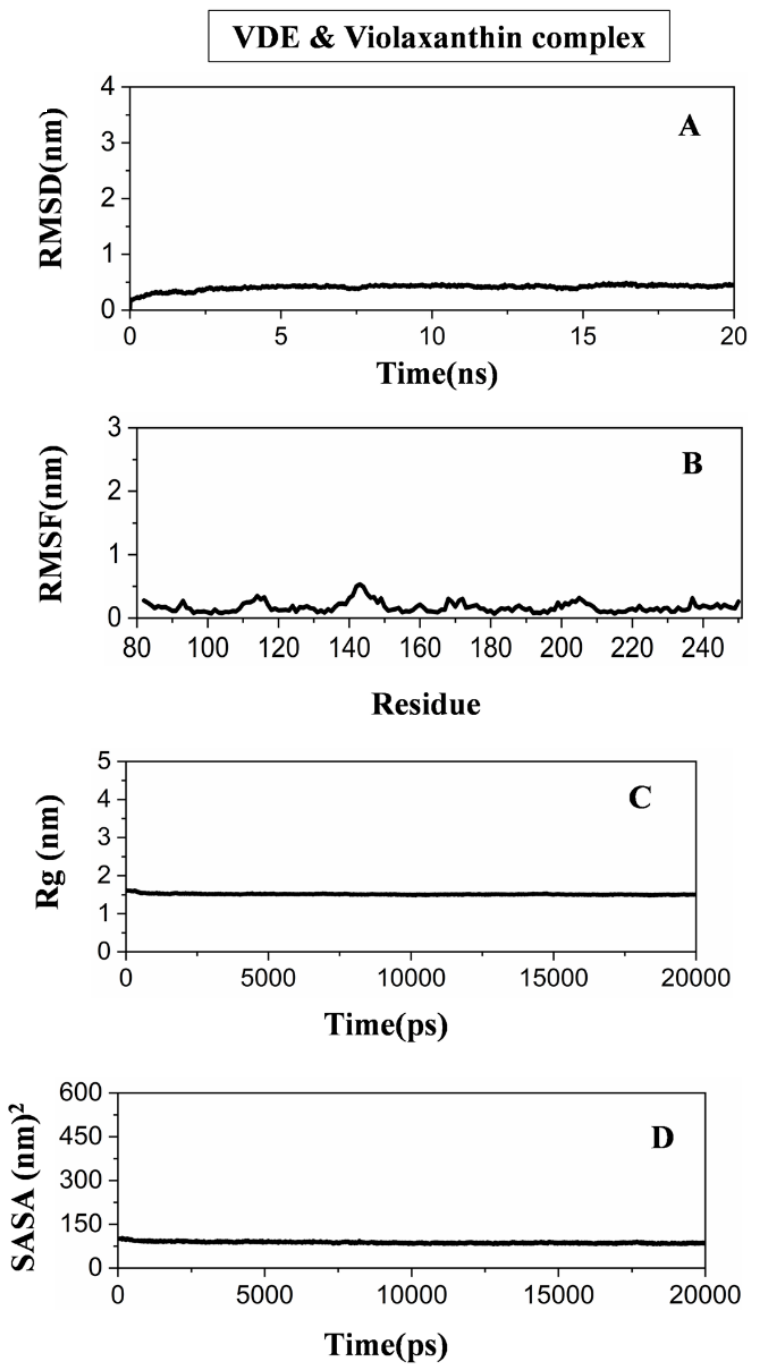
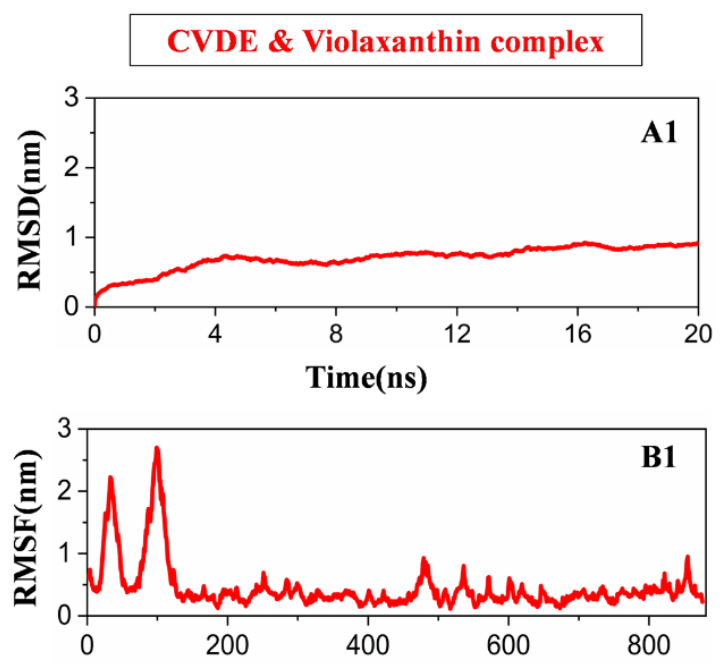

Residue
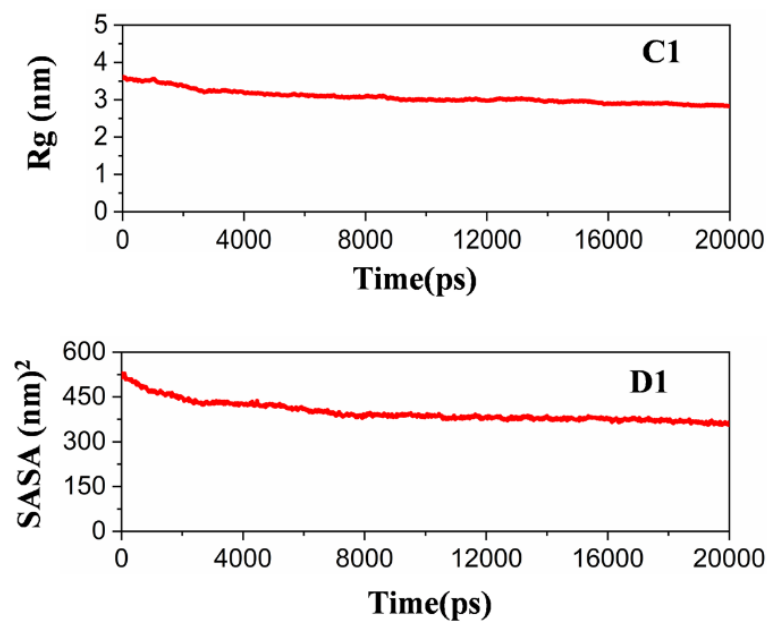

Figure 2: Plots of the root-mean-square deviation (RMSD) (A \& A1), root-mean-square fluctuation (RMSF) (B \& B1), radius of gyration $(\mathrm{Rg})(\mathrm{C} \& \mathrm{C} 1)$, and solvent accessible surface area (SASA) (D \& D1) of ascorbic acid (i) and violaxanthin (ii) complexes of VDE (black) and CVDE (red). 
bioRxiv preprint doi: https://doi.org/10.1101/2020.06.07.138495; this version posted June 8, 2020. The copyright holder for this preprint (which was not certified by peer review) is the author/funder, who has granted bioRxiv a license to display the preprint in perpetuity. It is made available under aCC-BY-NC-ND 4.0 International license.
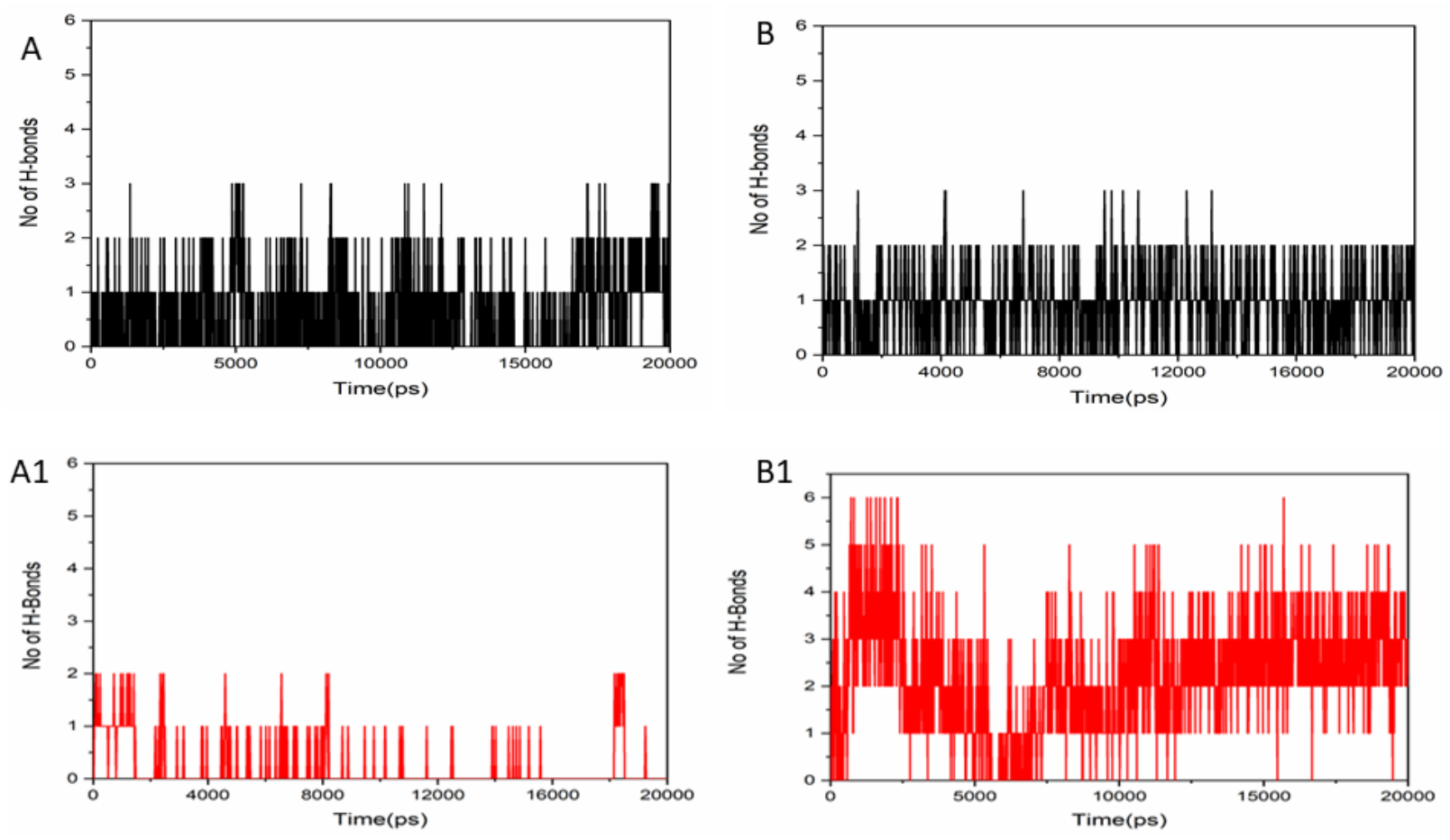

Figure 3: Plots of the average number of intermolecular h-bonds in the ascorbic acid (red) and violaxanthin (black) complexes of (A1) CVDE and (B1) VDE. 
bioRxiv preprint doi: https://doi.org/10.1101/2020.06.07.138495; this version posted June 8, 2020. The copyright holder for this preprint (which was not certified by peer review) is the author/funder, who has granted bioRxiv a license to display the preprint in perpetuity. It is made available under aCC-BY-NC-ND 4.0 International license.
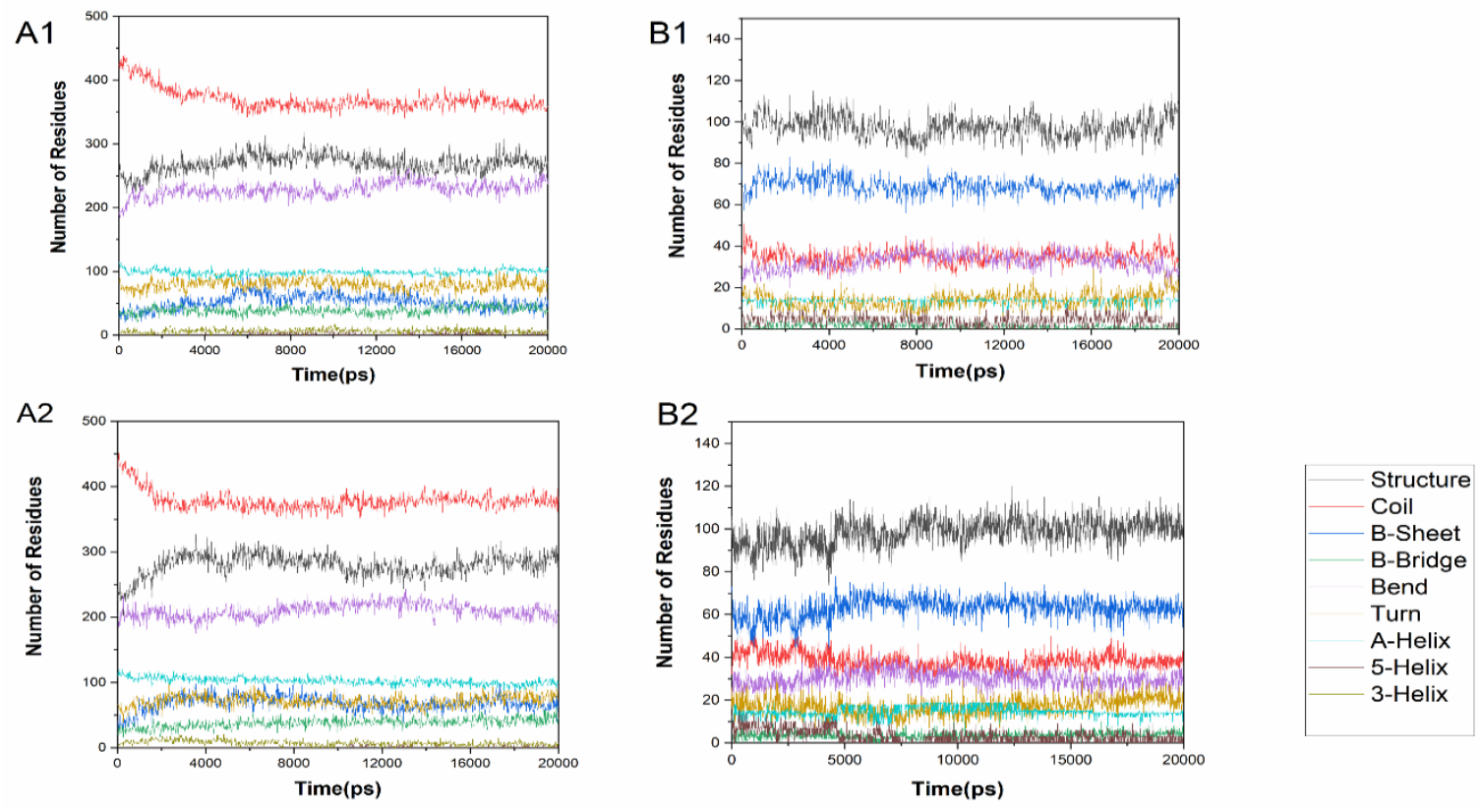

Figure 4: Secondary structure analysis of the CVDE and VDE complexes: (A1) VDE-ascorbic acid, (A2) VDEviolaxanthin, (B1) CVDE-ascorbic acid, and (B2) CVDE-violaxanthin. 

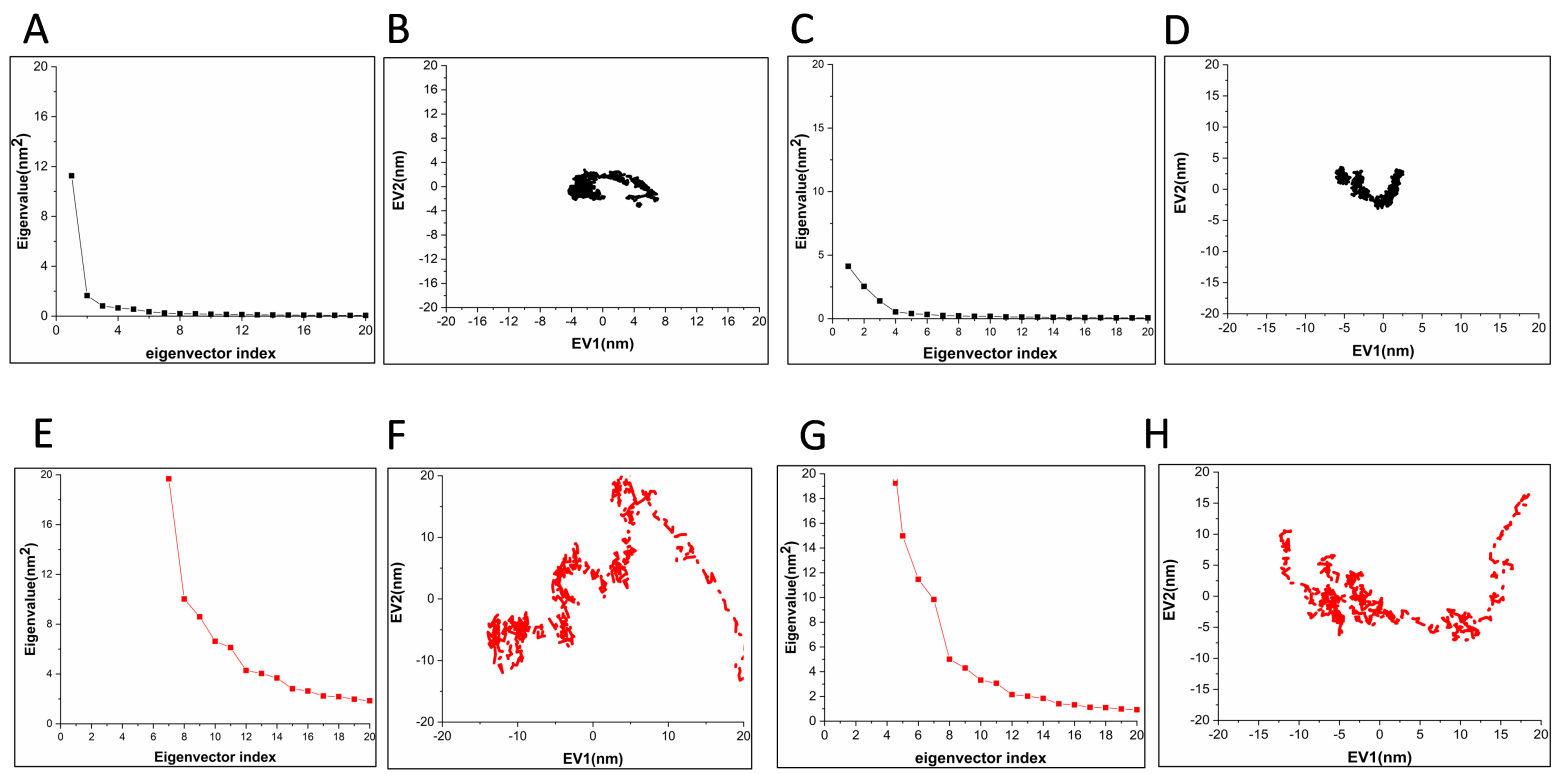

Figure 5: Principal component analysis of the CVDE and VDE complexes: plot of eigen values versus the corresponding eigen vector indices coming from the $\mathrm{C}_{\alpha}$ covariance matrix during MD simulations $(\mathrm{A}, \mathrm{C}, \mathrm{E}$, and $\mathrm{G})$ and the 2-D projection plot of the first two principal eigenvectors (B, D, F, and H). [Black: VDE complexes with both ascorbic acid (A \& B) and violaxanthin (C \& D); Red: CVDE complexes with both ascorbic acid (E \& F) and violaxanthin $(\mathrm{G} \& \mathrm{H})]$. 